\title{
Technology Self-organizing Ensembles of Intelligent Agents with Collective Synergetic Interaction
}

\author{
Evgeny Bryndin \\ Research Department, Research Center «Natural Informatics», Novosibirsk, Russia
}

\section{Email address:}

bryndin15@yandex.ru

\section{To cite this article:}

Evgeny Bryndin. Technology Self-organizing Ensembles of Intelligent Agents with Collective Synergetic Interaction. Automation, Control and Intelligent Systems. Vol. 8, No. 4, 2020, pp. 29-37. doi: 10.11648/j.acis.20200804.11

Received: October 27, 2020; Accepted: November 5, 2020; Published: November 11, 2020

\begin{abstract}
Technological collective ensembles are one of the new promising areas of artificial intelligence, which was formed on the basis of research results in the field of distributed computer systems, network technologies for solving problems, agents and systems with artificial intelligence and parallel computing. Ensemble of intelligent virtual agents can simultaneously interact with several external environments, conduct several dialogues and perform many skills, that is, can have collective knowledge and skills and behavior. The author, in this article, proposes a synergistic approach to the formation of technological ensembles of intelligent virtual agents. The synergistic approach created reference positions for the development of a new promising area of informatics. Synergistic approach establishes the relationships and from the image of conjugated system phenomena. It uses tools, approaches, and methodology to reasonably transfer the results of subsystem research, parts, and sections to their integral integrated and hierarchically superior system levels. The synergetic approach shows the most important role of resonant phenomena of the states of information systems. The synergistic principle provides a single basis for self-organization. The stage of self-organization occurs only in the case of positive feedback. Synergistic approach allows you to implement the self-organization of intelligent virtual agents of the technological ensemble. Technological self-organizing collective ensembles are able to interact with production teams, replace them for some time and even completely release them in various fields of professional activity. Technological collective ensembles can manage industries, make decisions in difficult changing circumstances and ensure safety in extreme conditions.
\end{abstract}

Keywords: Technological Ensemble, Intellectual Agents, Collective Skills and Behavior, Synergistic Self-organization, Changing Circumstances

\section{Introduction}

Agent technology becomes relevant in situations where rapid decision-making is required in a dynamic environment, such as risk or supply chain management, operational manufacturing, and supply chain management. This technology works well in a tiered and nodal real-time network when performing important operations and business processes. Companies can use process collective agents to select important transactions based on parameters such as time, delivery date, volume and minimum lot size.

The implementation of technological collective ensembles of intelligent agents will change the situation in the ecommerce market. The enormous potential of agent technology will be realized through direct interaction between digital business agents. Continuous interaction leads to the creation of very complex and dynamic systems that combine more agents. In a non-deterministic environment, each digital agent implements its functions, but together the collective agents realize a common task. When one of the agents is still busy, the others act to assume their functions and do not require further guidance. Agents perform certain actions, and by correctly combining these actions, you can simultaneously achieve the goals and achieve the required tasks. For example, the main task is to deliver goods, and the auxiliary goals are to increase speed and select the shortest routes. Agents define the actions that are necessary to achieve these goals, and then combine these actions according to business logic based on artificial intelligence [1-5].

Technological collective ensembles can automatically perform the entire cycle of e-commerce, from collecting information to deciding on a transaction. Adaptive decision- 
making by intelligent agents is achieved through collective knowledge as a result of analyzing the state of the business environment. For example, Christian Dannegar, Director of Technology at Living Systems, is developing collaborative commerce systems based on agent technology.

By 2050, to feed the growing population of the planet, it will be necessary to produce at least $70 \%$ more food. Smart agriculture and farming organizations will help technological collective ensembles to properly set up data collection, be able to analyze it and make operational decisions based on economic parameters [6]. Smart technologies in agriculture bring together four large clusters:

1. Exact agriculture (navigation systems, remote sensing (DZZ) and geographic information systems (GIS), differential application of fertilizers);

2. Agricultural robots (unmanned aerial vehicles, drones for tracking the state of fields and harvesting, smart sensor sensors).

3. AIoT platforms/AIoT applications (monitoring of data coming from sensors, technicians and other devices);

4. Big Data (analysis of data obtained from sensors for accurate forecasting and strategy);

5. AIoT platforms and applications.

AIoT platforms allow you to automate the entire cycle of agricultural operations for growing plants or animals. The mandatory components of such decisions are:

1. Peripheral equipment (sensors, sensors).

2. Communication channels (satellite communication GPS/GLONASS, LPWAN, LTE, 3G, GPRS, GSM).

3. AIoT platforms (web platforms for creating industry applications).

4. AIoT applications (applications for IT platforms, standalone applications for specific equipment).

5. The AIoT application generates logic for solving tasks, analyzes the received data flows and communicates with the user through the interface. Sometimes the AIoT platform and the AIoT application are one. The main application of the AIoT platform/applications is found in crop and soil monitoring for precision farming, monitoring of domestic and wild animals.

Farmers Edge (Canada) is the leader in providing innovative tools based on technology-based collective data management ensembles and precision farming services. The web and mobile application FarmCommand includes technologies for calculating the frequency of use of means, sampling and analysis of soil samples, meteorological summaries for farms, local communications and data transmission, daily satellite surveys, analysis of data obtained, forecasting, access to an integrated farm management platform and the real presence of specialists on the spot.

The article considers the main aspects of the functioning of intellectual agents, the collective interaction of intellectual agents, process self-organizing ensembles of intellectual agents, as well as the synergistic principle of the selforganization of the interaction of agents, the standard case of using an ensemble of intellectual agents and the means of implementing self-organizing ensembles of intellectual agents with collective synergistic interaction.

\section{Functioning of Intelligent Agents}

Intelligent agent works in a specific environment to achieve their goals. The environment is an activity field for the agent: it is subject to observations, the agent performs actions on it. An intelligent agent, achieving a goal in a certain environment, receives an award. The outcomes of certain possible actions are predicted using models. Intelligent agents use a physical and logical model of the environment. That is, they use both attributes and sets of entities, processes, relationships, etc.

An intelligent agent has a number of goals and permitted actions, his task will be to study what actions to take in each situation and in the conditions in which he finds himself. An intelligent agent tries to maximize the overall value of his goals over time, then for each particular observation he needs to choose actions that lead to the maximization of this value. To do this, the agent first: monitors the environment to determine the existing situation; predicts which of all possible actions will be the best; performs an action. Then he observes the influence of the action; Assess whether the impact has been good or bad and whether it has resulted in a goal; if not, determines whether it has brought it closer to the target or, conversely, removed it from the situation that was before the act was committed; Updates the prediction model to move the agent closer to the target. By repeating this process, the intelligent agent learns to take the most optimal actions in each situation. An intelligent agent solves two interrelated tasks - to learn and control. The work of the controller is to perform actions, behind it is the final decision on what action to take in each individual situation. The student's task is to create forecasts of the environment's reactions to the controller's actions. The student is a kind of adviser to the controller: he gives him recommendations on every possible action. The main goal of the agent is to receive more awards. This will depend only on how carefully we have determined the environment in which the agent lives.

The definition of the environment is based on its segmentation according to multiple user characteristics. To establish the value of possible actions with a large number of segments, you need to use mathematical models. Mathematical models can be used to approximate the relationship between the test characteristics and the predicted value of each action using predictive analysis. Predictive models help to obtain a generalized summary, on the basis of which the conclusion is made about the necessary actions. Using predictive models allows you to generalize or distribute knowledge among the observations obtained, which are not quite the same, but have similar features. This distribution of information allows us to make more accurate forecasts about a new customer audience, even one that we have not previously encountered. Predictive analysis optimizes the process if you determine in advance which user characteristics should be considered in decisionmaking at all. 


\section{Collective Interaction of Intelligent Agents}

The collective interaction of intellectual agents is characterized by the connection between coexisting agents, but also by the mutual transformation of the agents themselves and the relations between them. The main characteristics of their interaction are their orientation, selectivity, intensity and dynamics. Analysis of interaction between agents includes the following tasks:

1. identification of the situation of interaction of agents;

2. The allocation of core roles and their distribution among agents

3. determining the number and types of interacting agents;

4. Construction of a formal model of interaction;

5. Identify a set of possible agent behavior strategies

6. formation of many communicative actions.

The basic types of interaction between agents include cooperation, conflict, taking into account the interests of other agents, abandoning their interests in favor of others. Cooperation between agents can arise on a coercive basis (policy cooperation) or on a new voluntary relationship (situational cooperation). These two types of cooperation are often represented by the so-called con-tract form of cooperation, when the interaction of agents is regulated by a set of formal or informal agreements between them.

An intelligent agent has a limited set of knowledge he needs to achieve common goals. In this regard, he has to interact with other agents. At the same time, various situations are possible: a) the agent is able to perform for the delivery on his own; b) the agent can do without outside assistance, but cooperation will solve the problem in a more effective way; c) the agent is unable to solve the problem alone. Depending on the situation, agents chose the type of interaction and might show varying degrees of interest in cooperation.

Obligations are one of the tools to streamline the chaotic interactions of agents. They allow you to anticipate the behavior of other agents, predict the future and plan your own actions. The following groups of obligations can be distinguished: (a) obligations to other agents; b) the agent's obligations to the group; c) the group's obligations to the agent; d) obligation of the agent to himself. The formal representation of goals, obligations, desires and intentions, as well as all other characteristics, forms the basis of the mental model of the intel of the lectual agent, which ensures its motivated behavior offline. The listed reasons in different combinations can lead to different forms of interaction between agents:

1. Simple collaboration, which involves integrating the experience of individual agents (distribution of tasks, exchange of knowledge, etc.) without special measures to coordinate their actions;

2. Coordinated collaboration where agents are forced to coordinate their actions (sometimes involving a special coordinating agent) in order to leverage resources and expertise.

\section{Technological Self-organizing Ensembles of Intellectual Agents}

Technological collective ensembles use intellectual agents for the solution of one general difficult complex task. Agents make the action plans, being based already not only on the opportunities, but also on the basis of plans and intentions of other agents. For this purpose the ensemble needs to carry out a set of tasks:

1. formation of the joint action plans;

2. accounting of interests of partners of agents;

3. organization of negotiations on joint actions;

4. synchronization of joint actions;

5. recognition of need of cooperation;

6. choice of the suitable partner;

7. training in rules of conduct in collective;

8. decomposition of tasks and segregation of duties;

9. joint obligations, etc.

To organize an ensemble of agents with artificial intelligence in the likeness of human society, they are associated with systemic analysis, the theory of organizations, and the theory of management. An unresolved problem is the moral ethical basis of the organization, associated with the formation of concepts about the basic values and norms adopted in society. It is proposed to focus on models of regulatory behavior of agents. Problems of collective normative behavior are considered in system theory, in control theory and in game theory. The main idea of system analysis is to apply the decomposition of the original problem into simpler ones, from the solution of which a solution to the problem as whole can be found.

\subsection{Synergistic Principle of Self-organization of Interaction of Agents}

In ensembles, it is proposed to use the synergistic principle of interaction of agents with artificial intelligence for distributed re-creation of subtasks, as a strategy of number of collective behavior. The ensemble of agents with artificial intelligence is built as a self-organizing non-equilibrium thermodynamic system, which makes it possible to navigate in a difficult environment, deal with fuzzy tasks, adapt to changing conditions. They are able to solve complex problems on their own in conditions of uncertainty and high dynamics of change.

The main law of the ensemble organization is the law of synergy: the sum of the properties of the organized whole exceeds the sum of the properties available to each of the elements included in the whole separately. The most important feature of the system is the presence of qualities that are not reduced to the sum of the qualities of its constituent elements.

An important indicator of the sustainability of the organization as a holistic system is the nature of interaction with the environment. The self-regulation of the system consists in the fact that with internal or external influence on the system, some of its elements acquire dysfunctional 
properties, and in order to preserve themselves, the system seeks to neutralize these dysfunctions. The highest stage in the development of forms of regulation is management. The systems have a number of regulators subordinate to each other. Regulation as a process is a change in the relationship of elements of the system aimed at preserving it, and control is a process of transmitting information over communication channels, in which the functional nature of the properties of these elements is maintained and enhanced.

In the course of its existence, any organization undergoes certain changes, characterized by a change in its internal properties and characteristics, improvement in functioning, and a transition from one qualitative state to another.

The organization of the system is a complex and diverse process characterized by certain characteristics. In the formation of any system, firstly, communication between elements undergoes changes. Each element is functionally enriched. Secondly, the structural transformation of parts of the system, the limitation of some relationships and the expansion of others are assumed.

To bring elements into the system according to a given purpose, it is necessary to find a certain set of elements, reveal their functional properties, determine rational forms and methods for combining them. The unification is to create something qualitatively new, something that was not and could not be without this unification. To do this, you select characteristics or bases first to divide the elements, and then to join them into the system according to the law of proportionality. The law of proportionality defines the relationship between the organization of the system and the fact that there are certain quantitative and qualitative relations between each of the types of elements included in it, the elements themselves and between the characteristics of the elements. The law of proportionality determines the proportionality of parts combined as a whole, in which the effect of synergy is achieved.

To solve complex problems, a set of synergistic agents is offered. The ensemble constructed from there demonstrates the functions of an intellectual resonator assembled from components such as induction and deduction agents, synthesis, etc. Acting together as a single ensemble, intelligent agents are a powerful force with a high degree of intelligence, and solve many important life tasks in conditions of constantly changing conditions in the environment.

To develop the intelligence of the ensemble, you can create models of increasingly complex team interactions, including new classes of agents and protocols of their negotiations to achieve concessions, training from experience, etc. The higher the intelligence of each agent and the richer the possibilities of such communication between agents, the more complex and creative behavior the ensemble can demonstrate [7-15].

The synergistic principle directs to a new emerging:

1. analyze the change in the nature of the system interaction with the external environment;

2. Study the dynamics of the processes of system changes under the influence of the internal environment;

3. Study structures in an active state, capable of selfaction, spontaneous generation of new structures, selfmovement of the system;

4. take into account the life cycle phase of the system development.

The synergistic principle is based on the fact that information is the main driving factor in self-organizing systems of any nature. It determines the direction and main phases of the development of evolutionary processes, the structure and stability of the existence of natural, social, or man-made systems that arise.

Translating the content of bright events can produce a resonant process and their reactions and stimulate a mass response. In such conditions, order parameters are duplicated, structures close to them are excited, and as a result, simple systems are combined into complex structures. Structures fall into the same pace, begin to develop at equal speed. Involving structures in an agreed pace and rhythm encourages interaction according to general rules.

The synergistic principle provides a single basis for selforganization. The law of self-organization: the ability of the system to adapt to external disturbances, changing, if necessary, its structure and maintaining integrity. The selforganization of the technological ensemble is carried out by a set of intelligent adaptive agents that provide a given operability regardless of the operating conditions. The reasons for self-organization can be both external and internal.

Ensembles of agents with artificial intelligence on the synergistic principle of agent interaction implement selforganization. The intelligence of the ensemble manifests itself as an emerging property of a self-organizing system. There is a chain of agreed changes in the system of decisions by agents and spreads in the ensemble as a wave of approvals that determine the work of agents with artificial intelligence. Local interactions of agents form global structures, which in turn affect the behavior of the local agents that formed them and the intelligence of the ensemble.

The synergistic mechanisms of self-organization of technological ensembles of intelligent agents are basic for standardization in the application of ensembles in various fields.

\subsection{Standard Case Application of Ensemble of Intelligent Interoperable Agents}

Ensembles of agents with artificial intelligence are multiagent synergistic self-organizing systems that function according to the laws of development, synergy and selforganization. Intelligent agents use physical, informal and logical models of the environment. That is, they use both attributes and sets of entities, processes, relationships, etc.

Standard case Application of ensemble of intelligent interoperable agents defines parameters, characteristics, methods, human digital double models, knowledge, skills, behavior, images and other entities of intelligent virtual agent interaction (Table 1 - Table 7). Intelligent virtual agent 
interaction uses categorical method of utility and preference

Table 1. General.

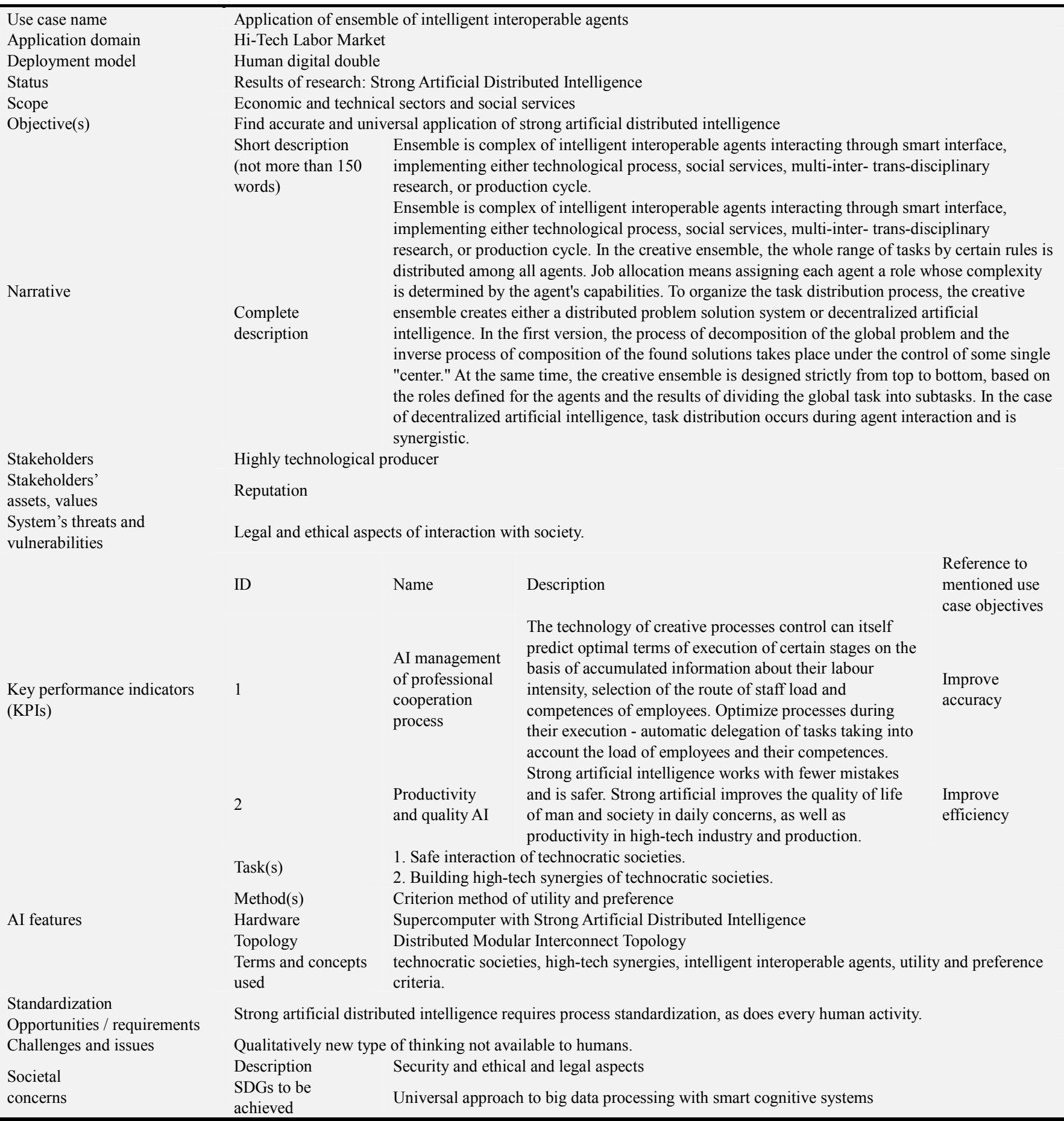

Table 2. Data

\begin{tabular}{ll}
\hline Data characteristics & \\
\hline Description & Strong Artificial Distributed Intelligence Data \\
Source & Model and technology of Strong Artificial Distributed Intelligence \\
Type & Strong \\
Volume (size) & Hi-Tech Labor Market \\
Velocity (e.g. real time) & Supercomputering Velocity \\
Variety (multiple datasets) & streams of multiple datasets \\
Variability (rate of change) & Retraining \\
Quality & High \\
\hline
\end{tabular}


Table 3. Process scenario.

\begin{tabular}{|c|c|c|c|c|c|}
\hline \multicolumn{6}{|c|}{ Scenario conditions } \\
\hline N. & Scenario name & Scenario description & Triggering event & Pre-condition & Post-condition \\
\hline 1 & Training & $\begin{array}{l}\text { Train a model (deep neural } \\
\text { network) with training data set }\end{array}$ & $\begin{array}{l}\text { Technological process raw data } \\
\text { set is ready }\end{array}$ & Formatting of data & Management of safety \\
\hline 2 & Evaluation & Expansion of the trained model & $\begin{array}{l}\text { Development of technological } \\
\text { thinking and behaviour }\end{array}$ & $\begin{array}{l}\text { Cognitive thinking } \\
\text { patterns and } \\
\text { psychological behaviors }\end{array}$ & $\begin{array}{l}\text { Meeting KPI } \\
\text { requirements is condition } \\
\text { of development }\end{array}$ \\
\hline 3 & Execution & Model and Technology Tooling & Interaction & Activization of Model & $\begin{array}{l}\text { Completion of } \\
\text { interaction }\end{array}$ \\
\hline 4 & Retraining & $\begin{array}{l}\text { Retrain a model with training } \\
\text { data set }\end{array}$ & $\begin{array}{l}\text { Certain period of time has passed } \\
\text { since the last training/retraining }\end{array}$ & $\begin{array}{l}\text { Additional data and } \\
\text { knowledge }\end{array}$ & $\begin{array}{l}\text { Combining Data and } \\
\text { Knowledge }\end{array}$ \\
\hline
\end{tabular}

Table 4. Training.

\begin{tabular}{|c|c|c|c|c|c|}
\hline Scenario name & Training & & & & \\
\hline Step No. & Event & Name of process/Activity & Primary actor & Description of process/activity & Requirement \\
\hline 1 & $\begin{array}{l}\text { Sample raw data set is } \\
\text { ready }\end{array}$ & Specification and classification & Manufacturer & Transform sample raw data & $\begin{array}{l}\text { Distributed AI } \\
\text { Software }\end{array}$ \\
\hline 2 & Completion of Step 1 & $\begin{array}{l}\text { Creating Set of Experimental } \\
\text { Data }\end{array}$ & Manufacturer & $\begin{array}{l}\text { Development of set of } \\
\text { experimental data through job } \\
\text { modelling }\end{array}$ & $\begin{array}{l}\text { Software of } \\
\text { modelling }\end{array}$ \\
\hline 3 & Completion of Step 2 & Model training & $\begin{array}{l}\text { AI solution } \\
\text { provider }\end{array}$ & $\begin{array}{l}\text { Train a model (deep neural } \\
\text { network) with experimental data } \\
\text { set created by Step } 2\end{array}$ & Big SD \\
\hline
\end{tabular}

Table 5. Evaluation

\begin{tabular}{|c|c|c|c|c|c|}
\hline Scenario name & Evaluation & & & & \\
\hline Step No. & Event & Name of process/Activity & Primary actor & Description of process/activity & Requirement \\
\hline 1 & $\begin{array}{l}\text { Completion of } \\
\text { training/retraining }\end{array}$ & Research & Manufacturer & $\begin{array}{l}\text { Train model (deep neural network) with } \\
\text { experimental data set created }\end{array}$ & Big SD \\
\hline 2 & $\begin{array}{l}\text { Completion of } \\
\text { Step } 1\end{array}$ & Identification & $\begin{array}{l}\text { AI solution } \\
\text { provider }\end{array}$ & $\begin{array}{l}\text { Based on data, detect execution using a deep } \\
\text { neural network trained in learning scenario }\end{array}$ & Big SD \\
\hline 3 & $\begin{array}{l}\text { Completion of } \\
\text { Step } 2\end{array}$ & Evaluation & Manufacturer & $\begin{array}{l}\text { Comparison of phase } 2 \text { results with human } \\
\text { performance }\end{array}$ & $\begin{array}{l}\text { Efficiency } \\
\text { and quality }\end{array}$ \\
\hline \multicolumn{2}{|c|}{ Output of evaluation } & Efficiency and quality & & & \\
\hline
\end{tabular}

Table 6. Execution.

\begin{tabular}{lllll}
\hline Scenario name & Execution & Primary actor & Description of process/activity & Requirement \\
\hline Step No. & Event & Name of process/Activity & Manufacturer & $\begin{array}{l}\text { Development of a set of } \\
\text { experimental data through job } \\
\text { modelling } \\
\text { Based on modified data train } \\
\text { model (deep neural network) with } \\
\text { experimental data set created }\end{array}$ \\
$\begin{array}{l}\text { Comparison of modeling } \\
\text { results with human }\end{array}$ & Research & Manufacturer & & \\
2 & Completion of Step 1 & Identification & & \\
Input of Execution & Modification \\
Output of Execution & Compatibility & & \\
\hline
\end{tabular}

Table 7. Retraining.

\begin{tabular}{|c|c|c|c|c|c|}
\hline $\begin{array}{l}\text { Scenario } \\
\text { name }\end{array}$ & Retraining & & & & \\
\hline Step No. & Event & $\begin{array}{l}\text { Name of } \\
\text { process/Activity }\end{array}$ & Primary actor & Description of process/activity & Requirement \\
\hline 1 & $\begin{array}{l}\text { Certain period of time has passed } \\
\text { since the last training/retraining }\end{array}$ & Research & Manufacturer & Additional data and knowledge & Completeness \\
\hline 2 & Completion of Step 1 & $\begin{array}{l}\text { Experimental } \\
\text { data set creation }\end{array}$ & Manufacturer & $\begin{array}{l}\text { Combining Data and Knowledge } \\
\text { Based on modified data train model } \\
\text { (deep neural network) with } \\
\text { experimental data set created }\end{array}$ & Compatibility \\
\hline 3 & Completion of Step 2 & Model training & AI solution provider & $\begin{array}{l}\text { Comparison of phase } 2 \text { results with } \\
\text { human performance }\end{array}$ & $\begin{array}{l}\text { Efficiency and } \\
\text { quality }\end{array}$ \\
\hline \multicolumn{2}{|c|}{ Specification of retraining data } & \multicolumn{4}{|c|}{ Retraining data set has to include recent data } \\
\hline
\end{tabular}




\subsection{Means of Implementing Self-organizing Ensembles of Intelligent Agents with Collective Synergistic Interaction}

To create intelligent agents, use the following description and implementation languages:

i. agent implementation languages and software;

ii. agent communication languages;

iii. languages describing the behavior of agents and the laws of the environment;

iv. presentation and knowledge management languages;

v. formalization languages and specifications of agents and multi-agent systems.

There are two approaches to designing agent interaction languages:

i. procedural - includes the exchange of procedural directives/commands.

ii. declarative, where the relationship is based on declarative instructions, type of definitions, assumptions, knowledge, etc.

Agent interaction tools include agent communication languages: FIPA ACL, KQML. The agent communication language enables the exchange of knowledge and information between agents. The FIPA ACL manages judgments, rules, and actions.

In the context of the practical construction of agents and ensembles, the main role is played by agent programming and communication tools. Communication languages (ACL, KQML) and agent coordination (AgenTalk) ensure consistent interaction between agents, circulation of information, transfer of service requests, implement negotiation mechanisms, support cooperation between agents aimed at achieving a common goal and, as a result, the formation of agent teams. These languages can be considered as multilevel structures, including the level of knowledge presentation, the level of negotiation or coordination, the level of communication strategies, etc.

For agent programming, the following can be used: universal languages (Java, C, Visual Basic, etc.), Knowledge Presentation Languages (SL, KIF), Negotiation and Knowledge Sharing Languages (KQML, AgentSpeak, April), script languages (Tcl/Tk, Python, Perl 5, etc.), specialized languages (TeleScript, COOL, Agent0, AgentK, etc.), symbolic languages and logical programming languages $(\mathrm{Oz}$, ConGolog, IMPACT, Dylog, Concurrent METATEM, Ehhf, etc.), as well as other languages and agent development tools.

One of the most well-known and well-established integrated development environments for intelligent software agents is the AgentBuilder of Reticular Systems, Inc. This tool consists of two main components, Toolkit and the execution system. The toolkit includes:

i. agent-based software development process management tools;

ii. means of analyzing the area of operation of the agent;

iii. tools for designing and developing networks from interacting agents; iv. tools for modeling the behavior of individual agents;

$\mathrm{v}$. tools for debugging and testing software agents.

AgentBuilder contains the Ummon tool for creating a selflearning agent. It also contains artificial intelligence methods for achieving human communication. The AgentBuilder Workbench provides developers with development tools and agent execution environments in the form of applications that display subject areas of tasks. The agent application runtime contains an agent execution processor. The processor uses efficient inference procedures by comparing agent behavior rules with agent beliefs determined by the current mental model and incoming messages. Based on the discussion, the processor performs certain actions related to the authority of the agent. The AgentBuilder toolkit is a modern and powerful tool for designing and implementing ensembles of intelligent agents.

JACK TM Intelligent Agents (JACK) is an agent-oriented development environment. The JACK Agent Programming Language offers the following features:

i. agent in JACK simulates intelligent entities;

ii. ability, which collects into a whole functional components (events, plans, many beliefs and other abilities), for use by their agents;

iii. an event to simulate situations and messages to which the agent must be able to respond;

iv. a plan that is designed to simulate a procedural description of how the agent manages this event (all actions taken by the agent are provided in advance and described in its plans);

v. many beliefs, for modeling the knowledge of the agent in the form of beliefs that adhere to the semantics of the closed or open world. This construction represents the agent's beliefs in the form of first-order relational tuples and ensures their logical consistency.

Agents work in such a way that agents process many plans and have access to descriptions of beliefs. Agents execute plans in event management tasks when they occur, comparing their beliefs when needed. These plans can initiate subtasks, which in turn can initiate their subtasks if the agent requires a time-consuming and complex response.

You have entered a new data structure named logical member, the value of which depends on the result of the request for multiple agent beliefs.

Ability to query multiple agents beliefs, using logical elements, by combining them to obtain the desired result. If the query succeeds, the logical element contains the desired value.

Agents created in JACK have the architecture inherent in intelligent agents. Thus, it is possible to model intelligent behavior in accordance with a theoretical model of agent architecture based on beliefs, desires and intentions. JACK intelligent agents are stand-alone software components that can exhibit intelligent behavior based on proactivity (targeting) and reactivity (event directed) on input signals. Each such agent has:

i. beliefs (this is his set of data on the world); 
ii. desires (a set of events to which it will respond and a set of goals to which it may wish);

iii. intentions (a set of plans that describe how it can manage emerging goals and plans). A plan set describes the steps an agent must take when a specific event occurs or wants to achieve a specific result.

With agents, you can realize the following properties associated with intelligent behavior:

i. sustainable targeting - agents focus on targets rather than on selected methods to achieve them;

ii. real-time context dependency - agents will monitor options that are applicable at each point in time and decide on follow-up actions based on conditions;

iii. asserting the real-time approach - the agent will ensure that it follows the chosen course of action as long as certain conditions continue to be true;

iv. concurrency - the ensemble of intellectual agents is multithreaded. If new goals and events occur, the agent is able to prioritize the demand for multitasking.

The self-organization of the interaction of intellectual agents is carried out according to criteria, rules and laws in a certain environment. Synergy between intelligent agents is achieved by setting up their self-organization to produce a result.

\section{Conclusion}

Currently, ensembles of agents with artificial intelligence are one of the most dynamic and promising areas in the field of self-organizing multi-agent systems. World-renowned Gartner estimates that by 2020 , multi-agent technologies will be the basis for more than $40 \%$ of all mobile applications. The development of ensembles of agents with artificial intelligence allows you to solve complex problems that previously could not be automated. The results of the decision give a quality comparable to a person's decision. Real-time event work is supported. It is possible to solve the problem in a dialogue with the user. Calculations can be easily paralleled to solve beyond complex problems. Agent ensembles with artificial intelligence have high openness, flexibility and efficiency, performance, scalability, reliability and survivability. They create a digital international ecosystem with telemedicine, remote monitoring and integration of diagnostic laboratories and drug delivery services [16]. In an epidemic, demand for online services, including medical services, breaks all records. The greatest economic effect of telemedicine consultations is observed in clinics where this service was provided before. The number of requests for online consultations is growing by $300-400 \%$ every week, while supply is still far behind demand. In quarantine mode, there is a massive cancellation by patients of their scheduled visits. Telemedicine consultations will help translate activities online and not only save business, but even increase the flow of patients. In regions with sad statistics on coronavirus, health care providers face a serious risk of this disease, and management faces the threat of a personnel crisis. Online consultations, in which there is no need for personal communication, are at the moment the optimal economic and epidemic solution for both patients, doctors, and managers. Even in a calm epidemic environment, the use of telemedicine technologies allows you to reduce the costs of both patients and the budget for consultations in federal medical centers by 2-4 times. Telemedicine counseling helps increase public access to health care for the public without being limited by federal quotas. Telemedicine based on ensembles of agents with artificial intelligence generally contributes to increasing the professionalism of medical personnel, reducing transportation costs and reducing the time spent in the hospital. The greatest development to date has been telemedicine in the USA, China, Great Britain. Almost half of the entire telemedicine market $(46 \%)$ is now in the United States. Telemedicine in the United States provides the patient with access to the specialists he needs 24 hours a day 7 days a week from his own house or office. Large telemedicine clinics are putting into practice diagnostic devices for laboratory results and breastfeeding devices integrated into the AppleHealth system. Medical equipment allows you to receive complete information about the patient's condition at home within a few minutes. Tyto is a multi-tool that combines the capabilities of a stethoscope that records the patient's heartbeat and breathing sounds and a high-resolution camera that removes the condition of the throat, ear passages and damage to the skin. MedWand - a device for diagnosing blood pressure, glucose and oxygen levels in the blood. Scanadu Scout - measures temperature, blood pressure and determines the level of oxygen in the blood. The basis of continuous patient care is the EHR systems - medical electronic archives. Most clinics are integrated into this health network. For example, CVS provides data access to over 40 health systems.

\section{References}

[1] Evgeniy Bryndin. Practical Development of Creative LifeSaving Strong Artificial Intelligence. Communications, Vol. 7 , No. 1, 2019. Pages: 31-39.

[2] Stewart Russell, Peter Norvig. Artificial Intelligence: Modern Approach. Williams, 2019. 1408 p. ISBN: 978-5-8459-1968-7.

[3] Wu Jun. Research on the Framework of Smart City Operating System Based on New ICTs. American Journal of Artificial Intelligence. Volume 4, Issue 1, June 2020, Pages: 36-41.

[4] Masloboev A. V., Langhans M. A multi-agent system for management information support of regional innovations. Scientific and Technical Journal of Information Technologies, Mechanics and Optics, 2018, vol. 18, no. 4, pp. 630-638.

[5] CRONEMBERGER F, GIL-GARCIA J R. Big data and analytics as strategies to generate public value in smart cities: proposing an integrative framework $[\mathrm{M}]$. Switzerland: Springer Nature, 2019.

[6] Fatima Kalna. A Meta-model for Diverse Data Sources in Business Intelligence. American Journal of Embedded Systems and Applications, Volume 7, Issue 1, June 2019, Pages: 1-8. 
[7] Evgeniy Bryndin. Development of Artificial Intelligence by Ensembles of Virtual Agents on Technological Platforms. COJ Technical \& Scientific Research. 2 (4). 2020. Pages: 1-8.

[8] Evgeniy Bryndin. Development of artificial intelligence by ensembles of virtual diversification agents. International Journal of Research in Engineering. Volume 2; Issue 1; 2020; Page 08-14.

[9] Oleg Vasylovych Moroz, Model of Self-organizing Knowledge Representation and Organizational Knowledge Transformation, American Journal of Artificial Intelligence. Vol. 4, No. 1, 2020, pp. 1-19. doi: 10.11648/j.ajai.20200401.11.

[10] Evgeniy Bryndin. Development of Artificial Intelligence by Ensembles of Virtual Agents with Mobile Interaction. Automation, Control and Intelligent Systems. Volume 8; Issue 1. 2020. Pages: 1-8.

[11] ISO/IEC JTC $1 / \mathrm{SC} 42 / \mathrm{WG} 4$ Use cases and applications Convenorship: JISC (Japan). 2019-12-23. https://isotc.iso.org/livelink/livelink/open/jtc1sc42wg4.

[12] Evgeniy Bryndin. Formation of Technological Cognitive
Reason with Artificial Intelligence in Virtual Space. Britain International of Exact Sciences Journal, Volume 2, Issue 2, May 2020. Page: 450-461.

[13] Evgeniy Bryndin. Standardization of Artificial Intelligence for the Development and Use of Intelligent Systems. Advances in Wireless Communications and Networks. Volume 6, Issue 1, 2020, pp. 1-9.

[14] Evgeniy Bryndin. Safe Interaction of Technocratic Societies Through Standardy Ensemble of Intellectual Virtual Agents. Control Science and Engineering. Vol. 4, Issue 1, 2020, pp. 815.

[15] Evgeniy Bryndin. Communicative-associative development of smart artificial intelligence by criteria with the help of ensembles of diversified agents. International Journal of Intelligent Information Systems. Volume 9, Issue 4, 2020.. pp. 24-34.

[16] Evgeniy Bryndin. Implementation of International Telemedicine network with Rapid Coronavirus Registration by Resonant Technology to Neutralize the Pandemic. Computational Biology and Bioinformatics. Vol. 8, Issue 2, 2020, pp. 29-35. 